\title{
Confidence Interval
}

National Cancer Institute

\section{Source}

National Cancer Institute. Confidence Interval. NCI Thesaurus. Code C53324.

A range of values for a parameter that may contain the parameter and the degree of confidence that it is in fact there. 